\title{
Dust emission from young outflows: The case of L1157
}

\author{
F. Gueth ${ }^{1}$, R. Bachiller ${ }^{2}$, and M. Tafalla ${ }^{2}$ \\ 1 Institut de Radioastronomie Millimétrique (IRAM), 300 rue de la Piscine, 38406 Saint Martin d'Hères, France \\ 2 IGN Observatorio Astronómico Nacional, Apartado 1143, 28800 Alcalá de Henares, Spain
}

Received 10 October 2002 / Accepted 20 February 2003

\begin{abstract}
We present new high-sensitivity $1.3 \mathrm{~mm}$ bolometer observations of the young outflow L 1157. These data show that the continuum emission arises from four distinct components: a circumstellar disk, a protostellar envelope, an extended flattened envelope - the dense remnant of the molecular cloud in which the protostar was formed -, and the outflow itself, which represents $\sim 20 \%$ of the total flux. The outflow emission exhibits two peaks that are coincident with the two strong shocks in the southern lobe of L 1157. We show that the mm continuum is dominated by thermal dust emission arising in the high velocity material. The spectral index derived from the new $1.3 \mathrm{~mm}$ data and $850 \mu \mathrm{m}$ observations from Shirley et al. (2000), is $\sim 5$ in the outflow, significantly higher than in the protostellar envelope ( 3.5). This can be explained by an important line contamination of the $850 \mu \mathrm{m}$ map, and/or by different dust characteristics in the two regions, possibly smaller grains in the post-shocks regions of the outflow. Our observations show that bipolar outflows can present compact emission peaks which must not be misinterpreted as protostellar condensations when mapping star forming regions.
\end{abstract}

\section{Introduction}

Mm and submm mapping of the dust thermal continuum emission has proven to be a powerful tool to study the density structure of low-mass protostellar condensations at different evolutionary stages (e.g. Motte et al. 1998; Chandler \& Richer 2000; Hogerheijde \& Sandell 2000; Shirley et al. 2000; Motte $\&$ André 2000). However, virtually all protostars are driving powerful molecular outflows (Bachiller 1996), which are much more extended than the protostellar envelope size (e.g. André et al. 1999). Strong interaction between the outflow and the envelope is observed in a few cases (e.g. L 1527, Motte \& André 2000). But besides such local perturbations, there is increasing observational evidence that the outflow itself can be responsible for (sub)mm continuum emission also at long distances from the driving source (Shirley et al. 2000; Chini et al. 2001). Since such continuum emission can be a valuable tracer of the outflow material, its detailed origin and structure clearly deserves further study.

The outflow located in the $\mathrm{L} 1157$ molecular cloud, at a distance of $440 \mathrm{pc}$, is one of the few which have been detected so far in the continuum submm and $\mathrm{mm}$ emission (Shirley et al. 2000; Chini et al. 2001). This outflow is driven by an extremely young Class 0 source (Gueth et al. 1997). The southern lobe consists of two large overlapping cavities, each one ended by a strong bow shock, which are misaligned due to the probable precession of the ejection axis (Gueth et al. 1996, 1998; Bachiller et al. 2001). The two shocks exhibit a rich molecular composition (Tafalla \& Bachiller 1995; Zhang et al. 1995; Avery \& Chiao 1996; Bachiller \& Pérez-Gutiérrez 1997)

Send offprint requests to: F. Gueth, e-mail: gueth@iram. fr which makes L 1157 the prototype of chemically active outflows (Bachiller et al. 2001). In this letter, we report new high-sensitivity bolometer observations of $\mathrm{L} 1157$ at $\mathrm{mm}$ wavelengths, which, in combination with previously published maps, provides basic information on the continuum emitting regions.

\section{Observations}

The observations were carried out in 1997 February and 1999 December, with the IRAM 30-m telescope. The detector was the MPIfR $1.3 \mathrm{~mm}$ bolometer array (MAMBO), which had 19 channels in 1997 and 37 channels in 1999. Each channel has an equivalent bandwidth of $\sim 70 \mathrm{GHz} .8$ individual on-thefly maps were obtained, with a different scanning direction for each coverage, from nearly perpendicular to almost parallel to the flow axis. The antenna beam width was $12^{\prime \prime}$. Skydips were observed every one to two hours to monitor the atmospheric zenith optical depth, which was found to be 0.1 to 0.4 . Flux calibration was achieved by observing Uranus. The final flux accuracy is estimated to be $\sim 30 \%$. The data were reduced with the IRAM package (NIC), using the EKH restoration algorithm. Fig. 1a presents the resulting image. The rms at the map center is $\sim 3.5 \mathrm{mJy} /$ beam.

Beltrán et al. (2003) obtained IRAM Plateau de Bure interferometric observations of the $1.3 \mathrm{~mm}$ continuum emission of the $\mathrm{L} 1157$ protostar. The bolometer map presented in this paper was used to derive the short-spacing information and thereby complement the interferometric dataset. The resulting map, which covers only the central region of the bolometer image, is shown in Fig. $1 b$. 


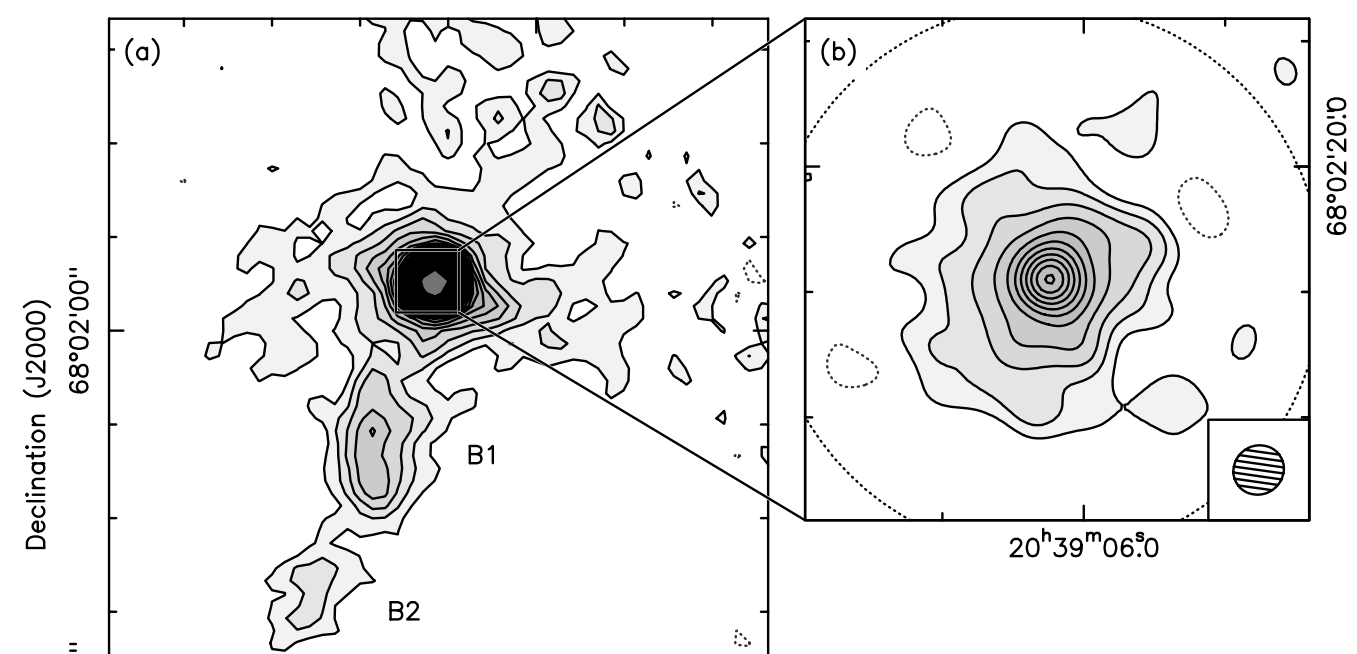

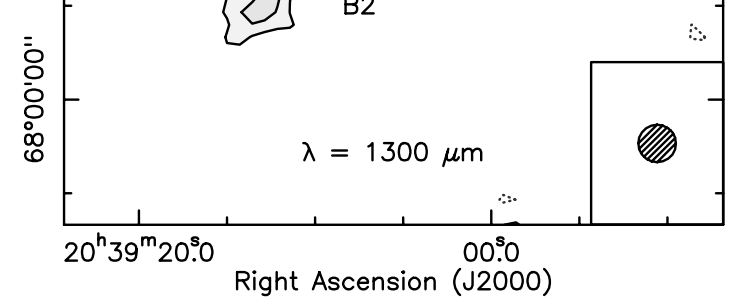

\section{Results}

\subsection{Four continuum components}

The data presented in Fig. 1 show that the mm continuum emission in L 1157 arises from four different components. Their properties are summarized in Table 1.

Central source. The interferometric observations (Fig. 1b) reveal the existence of a compact $\left(<1^{\prime \prime}\right)$ source, of $\sim 80 \mathrm{mJy}$, located at $20^{\mathrm{h}} 39^{\mathrm{m}} 06^{\mathrm{s}} .24,68^{\circ} 02^{\prime} 15^{\prime \prime} .6(\mathrm{~J} 2000)$. Obviously, this structure is closely associated to the protostar itself, and it is tempting to identify it with the thermal dust emission of a circumstellar disk. We refer to Beltrán et al. (2003) for a more detailed discussion.

Envelope. The central source is surrounded by an extended emission, that originates from the infalling (Gueth et al. 1997; Mardones et al. 1997) protostellar envelope. On the bolometer map, the peak flux density is $500 \mathrm{mJy} / \mathrm{beam}$, and the flux integrated in a circle of $40^{\prime \prime}$ diameter is $960 \mathrm{mJy}$. Assuming a typical dust temperature of $30 \mathrm{~K}$, the corresponding mass would be $\sim 2.1 M_{\odot}$ (Table 1). At the resolution of these observations (12"), the envelope is smooth and symmetric (the contour at half intensity is nearly round and has a diameter of $\sim 13^{\prime \prime}$, cf. Fig. 1a). However, the interferometric map (Fig. 1b) shows that the perturbation of the envelope by the outflow affects the internal structure of the envelope, which is no longer isotropic at that resolution, but extended along the flow axis.

Extended envelope. The L 1157 protostellar envelope is surrounded by an even more diffuse emission extended roughly east-west, i.e. approximately perpendicular to the flow axis (Fig. 1a). As shown in Fig. 2, this feature is also seen in the $850 \mu \mathrm{m}$ continuum map obtained by Shirley et al. (2000), as well as in the lower resolution $\mathrm{NH}_{3}(1,1)$ map obtained by Bachiller et al. (1993). It is also detected in quiescent emission of ${ }^{13} \mathrm{CO}$ (Gueth et al. 1997), $\mathrm{DCO}^{+}$, and $\mathrm{N}_{2} \mathrm{H}^{+}$(Bachiller et al. 2001). Very likely this structure is a dense remnant of the
Fig. 1. a) $1.3 \mathrm{~mm}$ bolometer map of L 1157. First contour and contour increment are $10 \mathrm{mJy} /$ beam $(3 \sigma)$. The angular resolution is $12^{\prime \prime}$. b) Interferometric map of the central region, from Beltrán et al. (2003). First contour and contour increment are $10 \mathrm{mJy} /$ beam $(3 \sigma)$. The angular resolution is $2^{\prime \prime}$. The dash circle indicates the interferometer primary beam $\left(22^{\prime \prime}\right)$.

Table 1. Properties of the four components of the $1.3 \mathrm{~mm}$ continuum emission in L 1157. The observed integrated fluxes are converted into masses assuming optically thin thermal dust emission, a uniform temperature, and a mass opacity $\kappa=0.01 \mathrm{~cm}^{2} \mathrm{~g}^{-1}$. The outflow emission has not been corrected for the molecular line contamination (see text).

\begin{tabular}{lcccc}
\hline \hline Component & Size & $\begin{array}{c}\text { Flux } \\
(\mathrm{mJy})\end{array}$ & $\begin{array}{c}\text { Temp. } \\
(\mathrm{K})\end{array}$ & $\begin{array}{c}\text { Mass } \\
\left(M_{\odot}\right)\end{array}$ \\
\hline Central source & $<1^{\prime \prime}$ & 80 & 30 & 0.15 \\
Envelope & $40^{\prime \prime}$ & 960 & 30 & 2.1 \\
Extended envelope & $140^{\prime \prime} \times 60^{\prime \prime}$ & 320 & 13 & 2.1 \\
Outflow & $40^{\prime \prime} \times 100^{\prime \prime}$ & 335 & $25-60$ & $0.3-0.9$ \\
\hline
\end{tabular}

molecular cloud in which the protostar was formed. The size of this envelope in the mm map is about $140^{\prime \prime} \times 60^{\prime \prime}$, its flux density is roughly constant, $\sim 12 \mathrm{mJy} /$ beam, and its total flux is $320 \pm 50 \mathrm{mJy}$ (after removing the $960 \mathrm{mJy}$ corresponding to the central source and its envelope). If the dust temperature is $13 \mathrm{~K}$ (as suggested by the $\mathrm{NH}_{3}$ observations of Bachiller et al. 1993), the mass of this extended structure is $\sim 2.1 M_{\odot}$.

Outflow. Besides the emission associated with the protostar vicinity, Fig. 1a shows that there is also an emission extending toward the south-east. A comparison with the $\mathrm{CO}(2-1)$ map of Bachiller et al. (2001), which has the same angular resolution, clearly shows that this emission is associated to the outflow (see Fig. 2): the continuum emission presents two main structures (labeled B1 and B2), whose positions and shapes correspond very well to those of the brightest $\mathrm{CO}$ emission. This is further demonstrated by the morphological agreement that exists between the mm continuum map and the $\mathrm{NH}_{3}(3,3)$ emission (Fig. 2) and the high-resolution $\mathrm{SiO}(2-$ 1) maps of Gueth et al. (1998). $\mathrm{The}^{\mathrm{NH}_{3}}$ and $\mathrm{SiO}$ emission trace shocks in molecular outflows. B1 does also coincide with the HH 375 Herbig-Haro object (Chini et al. 2001). We thus 


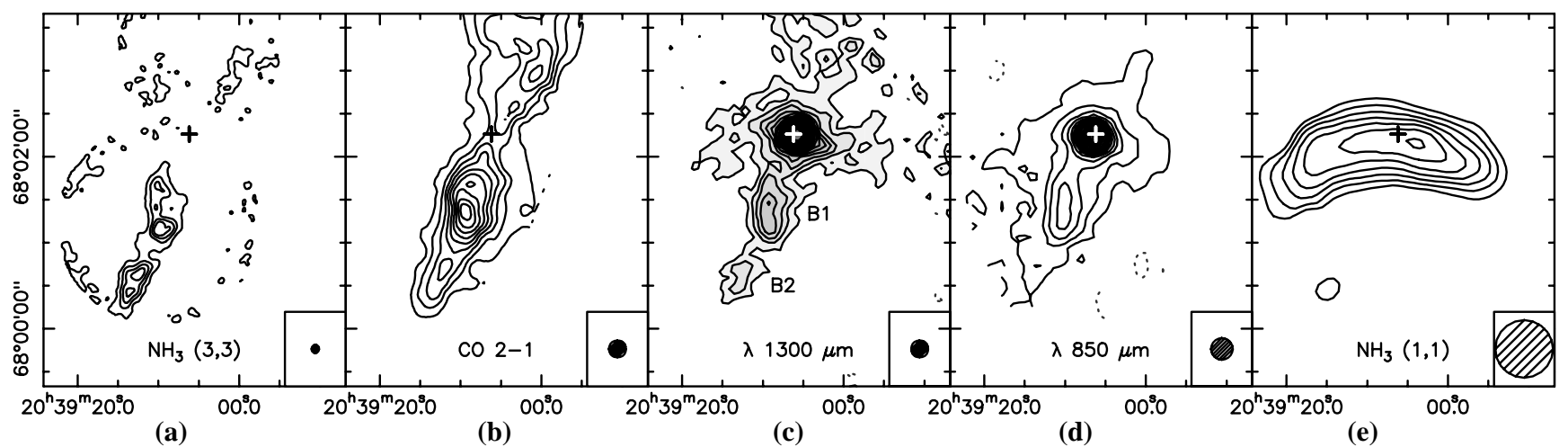

(a)

(b)

(c)

(d)

(e)

Fig. 2. Comparison of the $1.3 \mathrm{~mm}$ continuum emission map of $\mathrm{L} 1157$ with maps of other gas and dust tracers. a) $\mathrm{NH}_{3}(3,3)$ emission, from Tafalla \& Bachiller (1995). b) CO (2-1) emission, from Bachiller et al. (2001). c) $\lambda 1.3 \mathrm{~mm}$ continuum emission, see Fig. 1 . d) $\lambda 850 \mu \mathrm{m}$ continuum emission, from Shirley et al. (2000). e) $\mathrm{NH}_{3}(1,1)$ emission, from Bachiller et al. (1993).

conclude that significant emission arises from the shocked regions of the L 1157 outflow. The peak flux densities of B1 and B2 are $\sim 50$ and $\sim 25 \mathrm{mJy} /$ beam, respectively, and their integrated fluxes are 245 and $90 \mathrm{mJy}$.

\subsection{Origin of the outflow emission}

The broad bolometer bandwidth includes many molecular lines, which are quite strong in L 1157, and could thus create a spurious continuum emission. At the B1 position, the CO (2-1) line-integrated emission is $155 \mathrm{~K} \mathrm{~km}^{-1}$ (Bachiller et al. 2001), which corresponds to $\sim 8 \mathrm{mJy} /$ beam in the bolometer band, i.e. $16 \%$ of the detected emission. Other lines are weaker, but their sum can however be important: adding the integrated intensities of all lines (but CO) detected by Bachiller \& Pérez-Gutiérrez (1997) at B1 yields $110 \mathrm{~K} \mathrm{~km}^{-1}$. This puts the lines contribution to about $30 \%$ of the emission detected by the bolometer. This value could possibly be somewhat larger, since the line survey was not complete and not covering the full bolometer band.

Figure 2 also shows the SCUBA $850 \mu \mathrm{m}$ map obtained by Shirley et al. (2000). This map looks very similar to the $1.3 \mathrm{~mm}$ observations. From Hirano \& Taniguchi (2001), we can roughly estimate the $\mathrm{CO}(3-2)$ line-integrated emission to be $200 \mathrm{~K} \mathrm{~km}^{-1}$ at the $\mathrm{B} 1$ position, which corresponds to $\sim 20 \%$ of the bolometer measurement. Other lines must contribute and, as in the $1.3 \mathrm{~mm}$ case, could match the CO intensity and thus raise the line contribution to about $40 \%$.

We conclude that the lines contributions to the $1.3 \mathrm{~mm}$ and $850 \mu \mathrm{m}$ bolometer maps are significant, but are unlikely to account for the whole emission, which is thus dominated by continuum emission. A comparison of both maps reveals a spectral index in the range 3 to 5.5 (see below), which is typical for dust thermal emission, and thus suggests that any free-free emission is negligible in these images. Dust is actually thought to play a key role in the chemical processes taking place in shocked outflows (e.g. Schilke et al. 1997).

It is difficult to estimate the temperature of the dust in the outflow: the IRAS measurements (that would be needed to build the SED and then derive a temperature) do not have the angular resolution required to resolve the different components contributing to the L 1157 emission. Tafalla \& Bachiller (1995) used multiline $\mathrm{NH}_{3}$ analysis to derive outflowing gas temperatures of 60 to $80 \mathrm{~K}$. These values are upper limits to the temperature of the dust, which has a very short post-shock cooling time (e.g. Clark et al. 1986). Chini et al. (2001) suggested a dust temperature of $27 \mathrm{~K}$ for HH 375 (B1). From the measured $\mathrm{mm}$ fluxes, assuming optically thin emission and a temperature between 25 and $60 \mathrm{~K}$, we estimate that the mass of the blueshifted shocked regions is $\sim 0.3$ to $0.9 M_{\odot}$.

\subsection{Spectral index}

Figure 3 presents a map of the spectral index $\alpha$ computed between $850 \mu \mathrm{m}$ and $1.3 \mathrm{~mm}: \alpha=\log \left(S_{850 \mu \mathrm{m}} /\right.$ $\left.S_{1.3 \mathrm{~mm}}\right) / \log (1300 / 850)$. This image shows systematic variations across the mapped region. The lowest values of the index, in the range 3 to 4 , are obtained around the protostellar condensation. They are in good agreement with the average value $\alpha=3.4 \pm 0.3$ found by Shirley et al. (2000) in a sample of Class 0 sources. The highest values are in the range from 4.5 to 5.5, and are measured towards the outflow region.

Possible artifacts. A calibration error in one or both maps would translate into an incorrect scale for the spectral indexes, but would not introduce a position-dependent effect. However, line contamination of the bolometer measurements in the outflow region can affect the apparent spectral index by introducing an offset to the actual index of the continuum emission. Simple algebra shows that the values discussed in the previous section result in $\alpha$ being increased by 0.35 at $\mathrm{B} 1$, the position where the line contamination reaches its highest value. The variations of $\alpha$ at other positions are expected to be smaller. However, uncertainties on the line contamination, as well as possible calibration errors, make that this estimate of the $\alpha$ variation is only a rough approximation. In fact, the offset could be smaller (or even negative), if e.g. line contamination at $1.3 \mathrm{~mm}$ was underestimated. Inversely, if the line contribution at $850 \mu \mathrm{m}$ is higher, the offset can be larger: in order to introduce the observed 1.5 difference between the outflow and the protostellar envelope, a line/continuum ratio at $850 \mu \mathrm{m}$ of $\sim 2$ is required. This would imply that the $850 \mu \mathrm{m}$ map is essentially line emission, a possibility which - from the fluxes of the lines observed in the band - seems unlikely. 


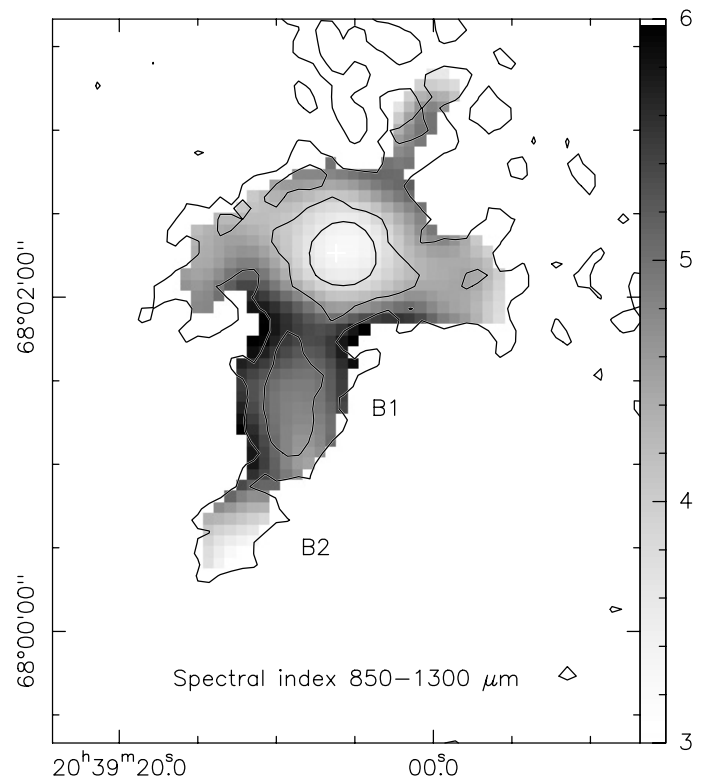

Fig. 3. Grey scale map of the spectral index around the blueshifted lobe of L 1157, computed between $850 \mu \mathrm{m}$ and $1.3 \mathrm{~mm}$. The value of the spectral index is indicated by the scale shown on the right. Three contours of the $1.3 \mathrm{~mm}$ emission map (Fig. 1) are also drawn for orientation. Before computing the index, we checked that both images agreed in position within $\sim 2^{\prime \prime}$, and smoothed them to the same resolution $\left(20^{\prime \prime}\right)$.

Dust properties. Besides the possible effects of the line contamination, a significant fraction of the variation in the spectral index has to be traced back to intrinsic properties of the continuum emission. For optically thin thermal dust emission, in the Rayleigh-Jeans limit, $\alpha=2+\beta$, where $\beta$ is the exponent in the dust opacity law $\kappa \propto v^{\beta}$. Hence, our observations would suggest that $\beta$ varies substantially from $\sim 1.5$ around the protostar to $\sim 3$ in the shocked regions ${ }^{1}$. The frequency variation of dust opacity is not well known, and depends on dust characteristics (see e.g. Visser et al. 1998, and references therein). Interestingly, a larger $\beta$ - as suggested in the flow points toward smaller dust grains (e.g. Krügel \& Siebenmorgen 1994) and/or different chemical properties (e.g. Pollack et al. 1994). Since the mm continuum emission in $L 1157$ arises from shocked regions, as shown by the morphological coincidences with shock tracers such as $\mathrm{NH}_{3}$ and $\mathrm{SiO}$, this result would be consistent with models predicting mantle evaporation (hence the rich chemistry observed in the shocks of L 1157) and even grain destruction (which may be the only way to release silicon and produce strong $\mathrm{SiO}$ emission, Schilke et al. 1997).

\section{Conclusion}

The observations presented in this letter demonstrate that the $\mathrm{mm}$ continuum emission in L 1157 originates from four

${ }^{1}$ Note however that the factor $h v / k$ is $\sim 11 \mathrm{~K}$ at $1.3 \mathrm{~mm}$ and $\sim 17 \mathrm{~K}$ at $850 \mu \mathrm{m}$, so the Rayleigh-Jeans approximation $(T \gg h v / k)$ fails e.g. in the extended envelope, where $T_{\text {dust }} \sim 13 \mathrm{~K}$ (if the dust is at the temperature indicated by the $\mathrm{NH}_{3}$ observations). In that case, $\alpha=$ $2+\beta+\gamma\left(T_{\text {dust }}\right)$, where $\gamma$ can easily be derived from the Planck function (see e.g. Visser et al. 1998). At $13 \mathrm{~K}, \gamma \sim-0.6$, but this correction is only 0.2 at $30 \mathrm{~K}$, and $\leq 0.1$ for $T_{\text {dust }} \geq 60 \mathrm{~K}$. Hence, this temperature effect cannot explain the observed variations of $\alpha$ across the L 1157 area, and one has to invoke variations of $\beta$. distinct components: the circumstellar disk, the infalling envelope, the dense remnant of the cloud in which the protostar has formed, and the outflow. The emission from the outflow represents $\sim 20 \%$ of the total flux at $1.3 \mathrm{~mm}$, and is dominated by dust thermal emission.

The spectral index computed between $1.3 \mathrm{~mm}$ and $850 \mu \mathrm{m}$ shows a significant difference between the protostellar envelope and the outflow emission. This could be related to an important line contamination of the $850 \mu \mathrm{m}$ map, and/or to variations of the dust opacity law index. In the latter case, this may be an indication of different dust properties, possibly of smaller grains in the outflow. Observations of additional bipolar flows are needed to check if such variations in the spectral index are a common phenomenon, and to investigate their origin.

Finally, we caution that large-scale $\mathrm{mm}$ and submm continuum mapping of molecular clouds (e.g. Motte et al. 1998) or of the vicinity of protostellar objects (e.g. Shirley et al. 2000) can potentially be confused by the presence of outflow emission, such as that from L 1157. Some emission knots could easily be misinterpreted as, e.g., starless clumps. The observations reported here demonstrate that emission from bipolar outflows can be important, and must be taken into account when interpreting the structure of mm maps of star forming regions.

Acknowledgements. The authors are grateful to the IRAM staff at Pico Veleta, to R. Neri for valuable help with the observations, and to Y. L. Shirley and N. J. Evans for providing a digital version of the $850 \mu \mathrm{m}$ map. This research have been partially supported by Spanish DGES grant AYA2000-927.

\section{References}

André, P., Motte, F., \& Bacmann, A. 1999, ApJ, 513, L57

Avery, L. W., \& Chiao, M. 1996, ApJ, 463, 642

Bachiller, R. 1996, ARA\&A, 34, 111

Bachiller, R., Martín-Pintado, J., \& Fuente, A. 1993, ApJ, 417, L45

Bachiller, R., \& Pérez-Gutiérrez, M. 1997, ApJ, 487, L93

Bachiller, R., Pérez-Gutiérrez, M., Kumar, M. S. N., \& Tafalla, M. 2001, A\&A, 372, 899

Beltrán, M. T., et al. 2003, in preparation

Chandler, C. J., \& Richer, J. S. 2000, ApJ, 530, 851

Chini, R., Ward-Thompson, D., Kirk, J. M., et al. 2001, A\&A, 369, 155

Clark, F. O., Laureijs, R. J., Chlewicki, G., et al. 1986, A\&A, 168, L1

Gueth, F., Guilloteau, S., \& Bachiller, R. 1996, A\&A, 307, 891

Gueth, F., Guilloteau, S., Dutrey, A., \& Bachiller, R. 1997, A\&A, 323, 943

Gueth, F., Guilloteau, S., \& Bachiller, R. 1998, A\&A, 333, 287

Hirano, N., \& Taniguchi, Y. 2001, ApJ, 550, L219

Hogerheijde, M. R., \& Sandell, G. 2000, ApJ, 534, 880

Krügel, E., \& Siebenmorgen, R. 1994, A\&A, 288, 929

Mardones, D., Myers, P. C., Tafalla, M., et al. 1997, ApJ, 489, 719

Motte, F., André, P., \& Neri, R. 1998, A\&A, 336, 150

Motte, F., \& André, P. 2000, A\&A, 365, 440

Pollack, J. B., Hollenbach, D., Beckwith, S., et al. 1994, ApJ, 421, 615

Schilke, P., Walmsley, C. M., Pineau Des Forets, G., \& Flower, D. R. 1997, A\&A, 321, 293

Shirley, Y. L., Evans, N. J., Rawlings, J. M. C., \& Gregersen, E. M. 2000, ApJS, 131, 249

Tafalla, M., \& Bachiller, R. 1995, ApJ, 443, L37

Visser, A. E., Richer, J. S., Chandler, C. J., \& Padman, R. 1998, MNRAS, 301, 585

Zhang, Q., Ho, P. T. P., Wright, M. C. H., \& Wilner, D. J. 1995, ApJ, 451, L71 